\title{
Lattice QCD study of static quark and antiquark correlations at finite $T$ via entanglement entropies
}

\author{
Toru T. Takahashi@ \\ National Institute of Technology, Gunma college, Maebashi, Gunma 371-8530, Japan \\ Yoshiko Kanada-En'yo \\ Department of Physics, Kyoto University, Sakyo, Kyoto 606-8502, Japan
}

(Received 30 November 2020; accepted 25 January 2021; published 11 February 2021)

\begin{abstract}
With the aim of clarification of color correlations among quarks, we investigate the color correlation between a static quark and an antiquark (static $q \bar{q}$ ) below and above the phase transition temperature $T_{c}$ through the entanglement entropy (EE). By a quenched lattice QCD calculation on an anisotropic lattice adopting the standard Wilson gauge action in the Coulomb gauge, we compute a reduced density matrix $\rho$ defined in the color space and the entanglement entropy $S_{\mathrm{EE}}$ constructed from $\rho$. The spatial volume is $L^{3}=24^{3}$, and the temporal extents are $N_{T}=12,13,14,15,16,18,20$ and 24, with a gauge coupling $\beta=5.75$ and a renormalized anisotropy $\xi=4.0$, which corresponds to temperatures between 180 and $370 \mathrm{MeV}$. From an analysis of $\rho$ and $S_{\mathrm{EE}}$, the color correlation between $q \bar{q}$ pairs is obtained as a function of the interquark distance $R$ and the temperature $T$. Below $T_{c}$, the $R$-dependence of the color correlation resembles that at $T=0$ : the quark's color correlation gradually decreases as $R$ increases due to the color screening by in-between gluons. Above $T_{c}$, due to the deconfinement phase transition, the color correlation among quarks is found to quickly disappear. Further, we investigate the color screening effect via the screening mass defined by $\rho$ and discuss the differences in the screening properties between the small and large $\mathrm{R}$ regions.
\end{abstract}

DOI: 10.1103/PhysRevD.103.034504

\section{INTRODUCTION}

Color confinement is one of the important nonperturbative features of quantum chromodynamics (QCD), which is the fundamental theory of the strong interaction. In the confinement phase, no colored state can be isolated in vacuum; hence, all the hadronic states are colorless. This color confining feature of QCD has been studied and confirmed using several approaches [1], and it may be explained by a linear potential among quarks arising in the large-separation limit (in the quenched QCD), which has been extensively investigated by analyses of interquark potentials for a variety of situations including multiquark systems [2-4]. At the same time, above the phase transition temperature $T_{c}$, the confining potential among quarks vanishes, and colored states are allowed, which has also been confirmed in lattice QCD calculations [5,6]. Such confining potential may be illustrated by the flux-tube formation among quarks. A color flux tube that has a

Published by the American Physical Society under the terms of the Creative Commons Attribution 4.0 International license. Further distribution of this work must maintain attribution to the author(s) and the published article's title, journal citation, and DOI. Funded by SCOAP. constant energy per unit length is formed among (totally color singlet) quarks, leading to the linearly rising potential $[2,7]$ in the large separation limit.

To cast light on the color structure of a confined quark pair, in Ref. [8], we computed the color correlation between a static quark and antiquark $(q \bar{q})$ pair by means of an entanglement entropy (EE) defined by the reduced density matrix $\rho$ in the color space at zero temperature. EEs defined by the reduced density matrix are known to quantify correlations (i.e., entanglements) between degrees of freedom in purely quantum systems, and they have been used in many physical systems [9-20]. Based on the EE as well as the reduced density matrix $\rho$, color correlation was obtained as a function of the interquark distance $R$ [8]. When totally color singlet quark and antiquark are located near each other, the $q \bar{q}$ pair forms a pure color singlet representation $|\mathbf{1}\rangle$ that yields the minimum $\mathrm{EE}$ (maximum color correlation). As the $q \bar{q}$ separation $R$ is enlarged, an octet color representation $|\mathbf{8}\rangle$ is randomly mixed in, and as a result, the quark color correlation is weakened, indicating that even in the confined phase a color screening between a $q \bar{q}$ pair occurs in the large $R$ region. If the color charge of the quark part and that of the gluon part are separately considered, such a color screening effect can be understood as a color leak from quarks to gluons. The color charge 
flows from a $q \bar{q}$ pair into in-between gluons (e.g., flux tubes) as quark separation is increased [21,22]. This color transfer from the quark part to the gluon part is observed as a screening effect on quark color correlation.

An investigation based on a color density matrix $\rho$ and an EE $S$ enables a model-independent analysis of the color structure of quarks. In this paper, we extend our previous work for zero-temperature systems in Ref. [8] to finitetemperature systems and investigate the color correlation between a $q \bar{q}$ pair below and above $T_{c}$. We define the reduced density matrix $\rho$ for a static $q \bar{q}$ pair in terms of color degree of freedom (d.o.f.) at finite temperatures and compute an EE $S$ from the reduced density matrix $\rho$. The reduced density matrix defined in the subspace of $q \bar{q}$ color configurations is computed by integrating the gluons' degrees of freedom, which can be achieved simply by averaging the density matrix components over gauge configurations. Through the analysis of $S$ and $\rho$ [8], we investigate the dependence of the color correlation on the interquark distance $R$ and the temperature $T$ below and above $T_{c}$.

In Sec. II, we present the formalism necessary to compute the reduced density matrix $\rho$ of the $q \bar{q}$ system and its EE $S$. The details of the numerical calculations and the ansatz for $\rho$ are shown in Sec. II. The results are presented in Sec. III, and Sec. IV is devoted to a summary and concluding remarks.

\section{FORMALISM}

\section{A. Reduced 2-body density matrix and entanglement entropy}

In this paper, we consider the SU(3) Yang-Mills theory on the lattice at finite temperature and follow the formalism shown in Ref. [8]. The reduced two-body density operator $\hat{\rho}(R)$ in a $q \bar{q}$ system with the interquark distance $R$ is defined as

$$
\hat{\rho}(R)=\operatorname{Tr}_{G}(|q(0) \bar{q}(R)\rangle\langle q(0) \bar{q}(R)|),
$$

where $|q(0) \bar{q}(R)\rangle$ denotes a quantum state in which an antiquark is located at the origin, while the other quark lies at $x=R . \operatorname{Tr}_{G}$ denotes the partial trace over the gluonic d.o.f. The reduced density matrix components $\rho(R)_{i j, k l}$ with $i, k(j, l)$ being the quark's (antiquark's) color indices are expressed as

$$
\rho(R)_{i j, k l}=\left\langle q_{i}(0) \bar{q}_{j}(R)|\hat{\rho}(R)| q_{k}(0) \bar{q}_{l}(R)\right\rangle .
$$

Here, $\rho(R)$ is an $m \times m$ square matrix with dimension $m=N_{c}^{2}$, and we directly compute $\rho(R)_{i j, k l}$ using lattice QCD techniques shown in the next section.

The von Neumann EE $S^{\mathrm{VN}}(R)$ for a $q \bar{q}$ pair separated by $R$ is constructed from the reduced density matrix $\rho(R)$ as

$$
S^{\mathrm{VN}}(R) \equiv-\operatorname{Tr} \rho(R) \log \rho(R) .
$$

Another kind of entropy, the Renyi EE [23] $S^{\text {Renyi- } \alpha}(R)$ of the order $\alpha$ is defined as

$$
S^{\text {Renyi- } \alpha}(R) \equiv \frac{1}{1-\alpha} \log \operatorname{Tr}\left(\rho^{\alpha}\right) .
$$

When computing $S^{\mathrm{VN}}$, one needs to diagonalize $\rho$ or approximate the logarithmic function, and to avoid such numerically demanding processes, throughout this paper, we adopt Renyi entropy [23] at $\alpha=2$ for EE. Note that in the limit when $\alpha \rightarrow 1$, it goes to von Neumann entropy as $S^{\text {Renyi- } \alpha} \rightarrow S^{\mathrm{VN}}$. Since the EEs, whose color indices are all contracted, are invariant under unitary transformations, they enable representation independent analysis of the $q \bar{q}$ pair color correlation.

Here, we briefly explain the relationship between the $q \bar{q}$ color correlation and the EE. Letting $Q$ denote the quark and antiquark's color d.o.f. and $G$ denote the gluon's color d.o.f., the $q \bar{q}$ pair's color correlation is defined in the subsystem $Q$. A pure state in the entire $Q+G$ system, which is created by a color singlet operator, can be written as

$$
\sum_{\alpha}|\alpha\rangle_{Q} \otimes|\bar{\alpha}\rangle_{G}
$$

Here, $\alpha$ denotes all the possible color representations of the $q \bar{q}$ pair, and $\bar{\alpha}$ represents the color representation of the gluon field which is determined so that $|\alpha\rangle_{Q} \otimes|\bar{\alpha}\rangle_{G}$ forms a color singlet state. When the quark's and antiquark's colors are strongly correlated forming a color-singlet combination $|\mathbf{1}\rangle_{Q}$ with no color charge leak from $Q$ to $G$, the subsystems $Q$ and $G$ are decoupled in the color space; hence, the whole state can be expressed as a direct product of $Q$ and $G$,

$$
|\mathbf{1}\rangle_{Q} \otimes|\mathbf{1}\rangle_{G}
$$

In this strongly correlated limit, the $\mathrm{EE} S^{\mathrm{EE}}$ goes to zero, since the two subsystems $Q$ and $G$ demonstrate no entanglement with each other. At the same time, when the $q \bar{q}$ pair's color charge flows into in-between gluons, and the $q \bar{q}$ color correlation decreases, the entire state cannot be written in a separable form, and $S$ becomes a positive finite value $(S>0)$.

\section{B. Ansatz for reduced density matrix $\rho_{i j, k l}(R)$}

In later sections, we perform an analysis of the EE as well as the density matrix $\rho$ based on the ansatz proposed in Ref. [8]. For the reader's convenience, here, we revisit the ansatz that reproduces the lattice QCD data well. We denote the density operator $\hat{\rho}_{s, s}$ for the quark and antiquark forming a pure color singlet state $|\boldsymbol{s}\rangle=\sum_{i}^{N_{c}}\left|\bar{q}_{i} q_{i}\right\rangle$ in the Coulomb gauge as 


$$
\hat{\rho}_{s, s}=|s\rangle\langle s| .
$$

The density operator $\hat{\rho}_{\boldsymbol{a}_{i}, \boldsymbol{a}_{i}}\left(i=1 \sim N_{c}^{2}-1\right)$ for $q \bar{q}$ in an adjoint state $\left|\boldsymbol{a}_{i}\right\rangle\left(i=1 \sim N_{c}^{2}-1\right)$ in color $\mathrm{SU}\left(N_{c}\right)$ QCD is expressed as

$$
\hat{\rho}_{\boldsymbol{a}_{i}, \boldsymbol{a}_{i}}=\left|\boldsymbol{a}_{i}\right\rangle\left\langle\boldsymbol{a}_{i}\right|\left(i=1 \sim N_{c}^{2}-1\right) .
$$

We assume that random color configurations of the $q \bar{q}$ pair, without any color correlation between quark and antiquark, are mixed with the maximally correlated color singlet component as the interquark distance $R$ is increased due to QCD interaction. In our previous paper, we demonstrated that this assumption is satisfied at $T=0$. In this ansatz, the density operator $\hat{\rho}$ can be written as

$$
\hat{\rho}_{\text {ansatz }}(R)=F(R) \hat{\rho}^{0}+(1-F(R)) \hat{\rho}^{\text {rand }} .
$$

Here, $F(R)$ represents the fraction of the original (maximally correlated) singlet state and $[1-F(R)]$ is that of the mixed (random) components. The maximally correlated state in which the $q \bar{q}$ pair forms $|\mathbf{1}\rangle$ gives $F(R)=1$, and at the random limit, when the quarks' colors are completely screened, it is represented by $F(R)=0$.

The explicit forms of $\hat{\rho}^{0}$ and $\hat{\rho}^{\text {rand }}$ are given as

$$
\begin{gathered}
\hat{\rho}^{0}=\hat{\rho}_{\boldsymbol{s}, s} \\
\hat{\rho}^{\mathrm{rand}}=\frac{1}{N_{c}^{2}} \hat{\rho}_{\boldsymbol{s}, s}+\frac{1}{N_{c}^{2}} \hat{\rho}_{\boldsymbol{a}_{1}, \boldsymbol{a}_{1}}+\frac{1}{N_{c}^{2}} \hat{\rho}_{\boldsymbol{a}_{2}, \boldsymbol{a}_{2}}+\cdots
\end{gathered}
$$

When $N_{c}=3$, this ansatz implies

$$
\left\{\begin{array}{l}
\rho(R)_{\mathbf{8}_{1}, \mathbf{8}_{1}}=\rho(R)_{\mathbf{8}_{2}, \mathbf{8}_{2}}=\ldots=\rho(R)_{\mathbf{8}_{8}, \mathbf{8}_{8}} \equiv \rho(R)_{\mathbf{8}, \mathbf{8}} \\
\rho(R)_{\alpha, \beta}=0 \quad(\text { for } \alpha \neq \beta)
\end{array}\right.
$$

The first relation should be satisfied due to the color SU(3) symmetry. The normalization condition $\operatorname{Tr} \rho=1$ is trivially satisfied in this ansatz as

$$
\rho(R)_{\mathbf{1}, \mathbf{1}}+\left(N_{c}^{2}-1\right) \rho(R)_{\mathbf{8 , 8}}=1 .
$$

In Ref. [8], the density matrix $\rho(R)_{\alpha, \beta}$ obtained by the lattice calculation was found to satisfy Eq. (12) with good accuracy for all $R$. Thus, we can perform analyses based on $\rho_{\text {ansatz }}(R)_{\alpha, \beta}$ instead of $\rho(R)_{\alpha, \beta}$, and, hereafter, we omit the subscript "ansatz" in $\rho_{\text {ansatz }}(R)_{\alpha, \beta}$.

Taking into account the normalization condition, the independent quantity at a given $R$ in this ansatz is only $\rho(R)_{\mathbf{8 , 8}}$ in Eq. (12), which we obtain as the averaged value of the lattice density matrix elements, $\rho(R)_{\mathbf{8 , 8}}=\frac{1}{N_{c}^{2}-1} \sum_{i} \rho(R)_{\mathbf{8}_{i}, \mathbf{s}_{i}}$. Then, we can compute the fraction $F(R)$ of the remaining correlated $q \bar{q}$ component as

$$
F(R)=\rho(R)_{\mathbf{1 , 1}}-\rho(R)_{\mathbf{8 , 8}}=1-N_{c}^{2} \rho(R)_{\mathbf{8}, \mathbf{8}} .
$$

\section{Lattice QCD setup}

We define a Polyakov line at the spatial point $\boldsymbol{x}$ as

$$
P_{i j}(\boldsymbol{x}) \equiv\left[\prod_{\tau=0}^{N_{\tau}-1} U_{4}(\boldsymbol{x}, \tau)\right]_{i j},
$$

where $i, j$ denotes the color index, and $\tau$ is the temporal position. $U_{4}(\boldsymbol{x}, \tau)$ is a Euclidean temporal link variable at $(\boldsymbol{x}, \tau)$.

With a normalization factor $N$ determined such that

$\operatorname{Tr}\left\langle\frac{1}{N} P_{i j}^{\dagger}(0) P_{k l}(R)\right\rangle=\sum_{i j}\left\langle\frac{1}{N} P_{i j}^{\dagger}(0) P_{i j}(R)\right\rangle=1$,

we obtain $\rho(R)_{i j, k l}$ whose trace is unity $(\operatorname{Tr} \rho(R)=1)$ as

$$
\rho(R)_{i j, k l}=\left\langle\frac{1}{N} P_{i j}^{\dagger}(0) P_{i j}(R)\right\rangle .
$$

Once we obtain $\rho(R)$, Renyi entropy of the order $\alpha$ as a function of $R$ can be obtained as

$$
S^{\text {Renyi- } \alpha}(R)=\frac{1}{1-\alpha} \log \operatorname{Tr}\left(\rho(R)^{\alpha}\right) .
$$

In this study, we adopt the standard Wilson gauge action that exhibits a temporal anisotropy, and perform quenched calculations for reduced density matrices of static $q \bar{q}$ systems at finite temperatures. The gauge configurations are generated on the spatial volume $L^{3}=24^{3}$, with the gauge coupling $\beta=5.75$ and the renormalized temporal anisotropy $\xi=4.0$ [24]. This leads to a spacial cutoff $a_{\sigma}^{-1}$ of $1.1 \mathrm{GeV}$ and a temporal cutoff $a_{\tau}^{-1}$ of $4.4 \mathrm{GeV}$ [25]. The temporal extents $N_{\tau}$ employed in this work are $N_{\tau}=12$, $13,14,15,16,18,20$ and 24 , which correspond to the temperatures of 183-367 MeV. All the gauge configurations are gauge-fixed with the Coulomb gauge condition.

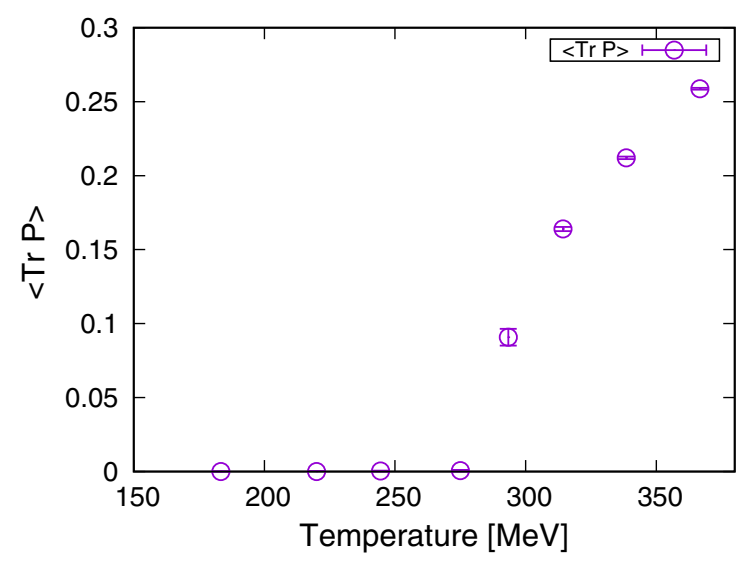

FIG. 1. The expectation value of a Polyakov loop $\langle\operatorname{Tr} P\rangle$ plotted as a function of the temperature $T$. 
The deconfinement transition was investigated using an anisotropic lattice and found to occur at $T=T_{c}=$ $285 \mathrm{MeV}$ [26]. Since a precision study of the transition temperature $T_{c}$ is not within the scope of the present work, we conclude this section simply by showing the expectation value of a Polyakov loop $\langle\operatorname{Tr} P\rangle$, an order parameter in quenched QCD, obtained in our setup in Fig. 1.

\section{LATTICE QCD RESULTS}

\section{A. Entanglement entropy}

We adopt Renyi entropy of $\alpha=2$ for the evaluation of the color correlation between $q \bar{q}$ pairs. The $S^{\text {Renyi-2 }}(R)$ is obtained by taking the logarithm of the trace of the squared reduced density matrix $\rho(R)$ as

$$
S^{\text {Renyi-2 }}=-\log \operatorname{Tr}\left(\rho(R)^{2}\right) .
$$

Taking into account the normalization condition $\operatorname{Tr}(\rho(R))=1$, the maximum of $S^{\text {Renyi-2 }}$ is reached when all the $N_{c}^{2}$ diagonal elements are equal to $1 / N_{c}^{2}$ in the diagonal representation of $\rho(R)$. Based on the representation invariance of $S$, the maximum value of $S$ is

$$
\max \left[S^{\text {Renyi-2}}(R)\right]=2 \log N_{c} .
$$

In the upper panel in Fig. 2, $S^{\text {Renyi-2 }}(R)$ values calculated from the $\rho(R)$ obtained on the lattice are plotted. At small $R$ values, $S^{\text {Renyi-2 }}(R)$ exhibits smaller values indicating that the $q \bar{q}$ pair forms a pure color singlet state and is strongly correlated at $R \rightarrow 0$. $S^{\text {Renyi-2 }}(R)$ rises with increasing $R$ and reaches a maximum value $\left(2 \log N_{c}\right)$ at a large $R$ value, indicating that the $q \bar{q}$ color configuration approaches a random configuration without color correlation in the large $R$ range. This tendency is observed at all temperatures.

Below $T_{c}=285 \mathrm{MeV}, S^{\text {Renyi-2 }}(R)$ values are more or less similar to each other, indicating that the thermal effect on the color correlation in the confined phase is not apparent in the investigated temperature range. At $T=T_{c}$, the $q \bar{q}$ color correlation is quickly quenched due to the deconfinement transition, which can be observed as a sudden increase in the $S^{\text {Renyi-2 }}(R)$ across $T_{c}$. Above $T_{c}, S^{\text {Renyi-2 }}(R)$ for $R \geq 0.6 \mathrm{fm}$ take almost the maximum value and reveal a vanishing $q \bar{q}$ color correlation. In contrast, $S^{\text {Renyi-2 }}(R)$ at $R<0.6 \mathrm{fm}$ have a $T$ dependence, and it shows that the color correlation still exists between the $q \bar{q}$ pair at short distances even in the deconfined phase.

To take a brief look at the thermal effect on the $q \bar{q}$ color correlation, we plot $S^{\text {Renyi-2 }}$ as a function of $R T$ in the lower panel in Fig. 2. It is found that the EEs above $T_{c}$ $(=285 \mathrm{MeV})$ lie on a common curve, except for those for $T=293 \mathrm{MeV}$ just above $T_{c}$, which is considered to be transient region. This indicates that the color screening effect above $T_{c}$ caused by thermal fluctuations depends on $R$ normalized by $1 / T$, which would be related to the Debye screening length in the deconfined phase. We discuss the
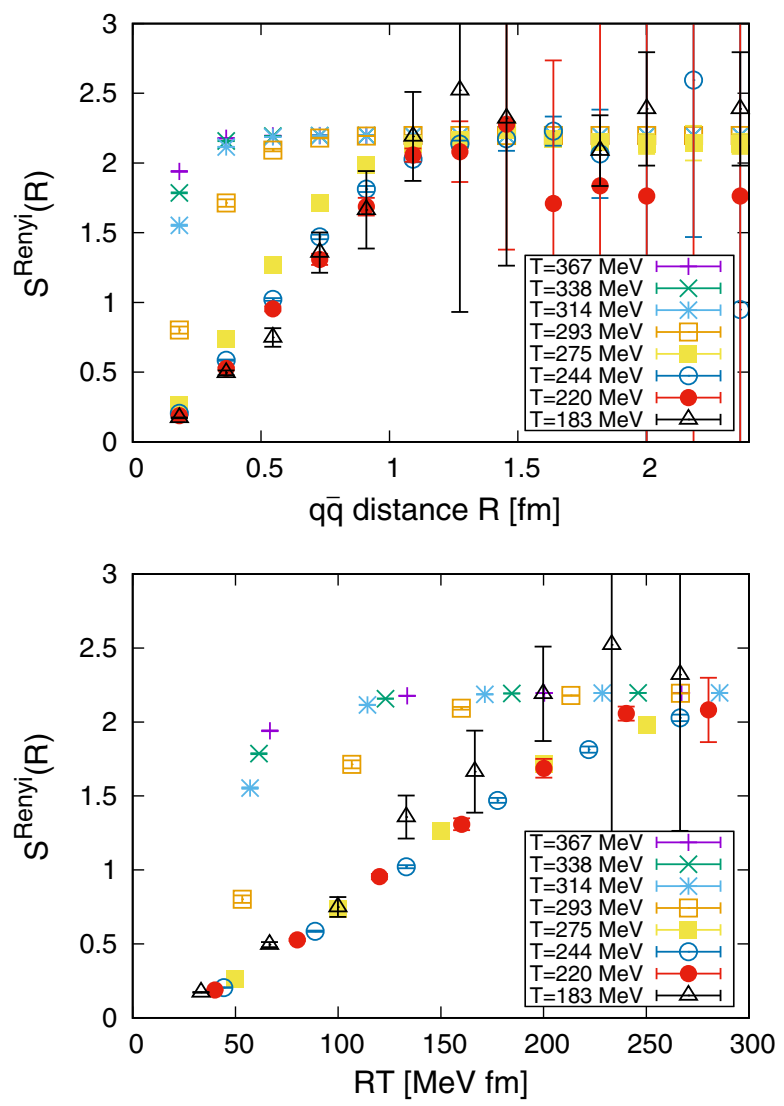

FIG. 2. The upper panel shows $S_{\text {lattice }}^{\text {Reni-2 }}(R)$ at each $T$ obtained from the reduced density matrix $\rho(R)$ plotted as a function of the interquark distance $R$, and the lower panel indicates $S_{\text {lattice }}^{\text {Renyi-2 }}(R)$ plotted against $R T$.

color screening mass in a quantitative way in later sections. It is interesting that the EEs obtained below but near $T_{c}$ also lie on a common curve.

To investigate the $T$ dependence of EE in more detail at each distance $R$, we plot the EEs as a function of $T$ (Fig. 3). Below $T_{c}$, the $S^{\text {Renyi-2 }}(T)$ values for $R<0.6 \mathrm{fm}$ are less than $30 \%$ of the maximum value, revealing the strong

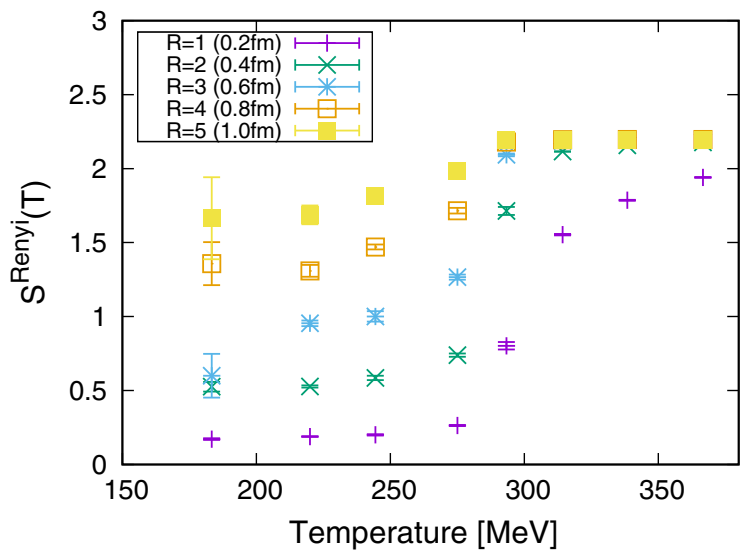

FIG. 3. $S_{\text {lattice }}^{\text {Renyi-2 }}(R)$ at each $R$ plotted as a function of the temperature $T$. 
correlation between a $q \bar{q}$ pair. For $R \geq 0.6 \mathrm{fm}, S^{\text {Renyi-2 }}(T)$ reach greater than $50 \%$ of the maximum value, indicating a reduced color correlation due to color leak to the inbetween flux-tube. At $T=T_{c}$, the EEs for all $R$ increase suddenly, and the $q \bar{q}$ color correlation is mostly lost after the deconfinement phase transition. Taking into account that the quarks' color correlation is closely related to the surrounding gluon configuration, the increase in $\mathrm{EE}$ at $T=T_{c}$ reflects a drastic modification of the gluon configuration at the phase transition. Above $T_{c}$, the EEs for $R<0.6 \mathrm{fm}$ do not reach the maximum value at $T$ values close to $T_{c}$, continuing to grow as $T$ increases. This indicates that, even in the deconfined phase, a thermal effect on the $q \bar{q}$ color correlation still exists. This behavior can be understood based on the thermal effect on color screening by the gluon medium, which will be discussed in the context of the "color screening mass" in a later section.

While EEs show a sudden increase at the phase transition point for any $R$, the $\mathrm{EE}$ at the shortest distance $(R=0.2 \mathrm{fm})$ shows an especially clear change and behaves like an order parameter, the expectation value of a Polyakov loop (Fig. 1). In the limit $R \rightarrow 0$, the $q \bar{q}$ pair is dominated by a strongly correlated color singlet state $|\mathbf{1}\rangle$ and no color leak to the system occurs due to the color confinement, which leads to the small $\mathrm{EE}$, amounting to just $10 \%$ of the maximum value at $T<T_{c}$. After the deconfinement phase transition, $q \bar{q}$ pair is also able to form a color nonsinglet (octet) state $|\mathbf{8}\rangle$ even in the case of $R \rightarrow 0$. In this case, the color charge largely spread into the surrounding gluon field and the color correlation is lost. This sudden mixture of the color octet $q \bar{q}$ states leads to significant enhancement of EE above $T_{c}$.

It should be noted that below $T_{c}$, the $T$ dependence of $S_{\text {lattice }}^{\text {Reni-2 }}(R)$ is small for all $R$ values, but the $S_{\text {lattice }}^{\text {Renyi-2 }}(R)$ is not entirely constant, as can be seen in Figs. 2 and 3. This may indicate a thermal influence on the color correlation in the confined phase. For a clarification of the thermal effects in the confined phase, a further detailed analysis with high statistics is needed.

\section{B. Analysis using an ansatz}

To investigate the $q \bar{q}$ color correlations in more detail, we compute $F(R)$ defined in Sec. II B by adopting the ansatz Eq. (14). The color screening effect by the inbetween gluon field is encoded in $F(R)$, which is the only parameter in this ansatz that indicates the fraction of the color-singlet component of the $q \bar{q}$ pair. $F(R)=1$ for a pure color singlet state (the maximally correlated $q \bar{q}$ pair), and $F(R)=0$ for the random color state of the $q \bar{q}$ pair (the uncorrelated $q \bar{q}$ pair).

In Fig. $4, F(R)$ at each $T$ is plotted as a function of the interquark distance $R$. The lower panel in Fig. 4 shows the logarithmic plot of $F(R)$. Below $T_{c}, F(R)$ appears to change its slope around $R=0.6 \mathrm{fm}$ at each $T$, as can be seen in the lower panel. $F(R)$ seems to linearly decrease in


FIG. 4. The upper panel shows $F(R)$ at each temperature plotted as a function of the interquark distance $R . F(R)$ monotonously decreases, approaching zero. The lower panel shows a logarithmic plot of $F(R)$.

the short-distance region $(R<0.6 \mathrm{fm})$, while decaying exponentially at larger $R$ values $(R \geq 0.6 \mathrm{fm})$. This change in $R$ dependence might indicate flux-tube formation between $q \bar{q}$ pairs at $R \geq 0.6 \mathrm{fm}$. In other words, a flux tube is generated between $q \bar{q}$ pairs with a large separation $R$, and the in-between gluons forming the flux tube screen the quarks' color, leading to exponential decay of the correlation. At the same time, in the small $R$ region, the quarks' color is not screened by surrounding gluons, which instead produce Coulomb-type interactions. To investigate this in detail, here, we analyze the damping factor in these two distinct regions of $R$, defining a "color screening mass" in the next section. Above $T_{c}, F(R)$ appears to exponentially decay over all $R$ regions. This damping behavior can be understood as a medium effect in the deconfined phase, where a $q \bar{q}$ pair feels a screened Yukawa-type potential over the entire $R$ range; hence, $F(R)$ provides exponential damping with a constant damping factor.

\section{Color screening mass}

The exponential damping of the $q \bar{q}$ correlation $F(R)$ indicates the color screening effects of in-between gluons. We fit $F(R)$ with an exponential function as 


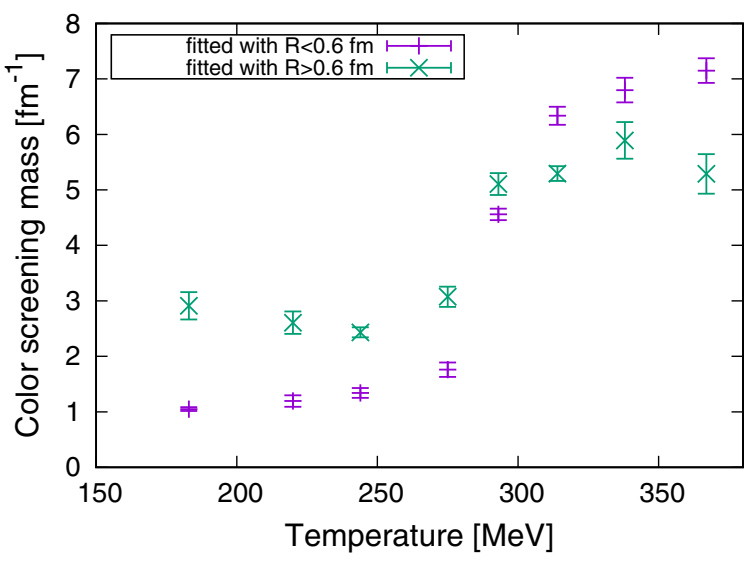

FIG. 5. The color screening masses $B$ fitted in the range of $0 \leq R \leq 0.6$ and $R \geq 0.6 \mathrm{fm}$ plotted as a function of $T$. The fit was performed with the functional form $F(R)=A \exp (-B R)$.

$$
F(R)=A \exp (-B R)
$$

and extract the "color screening mass" $B$. In particular, since the behavior of $F(R)$ appears to qualitatively change at $R \sim 0.6 \mathrm{fm}$, we adopt two distinct fit ranges $(R<0.6 \mathrm{fm}$ and $R \geq 0.6 \mathrm{fm}$ ). In Fig. 5, the color screening masses $B_{<}(T)$ and $B_{>}(T)$ extracted in the two ranges $R<0.6$ and $R \geq 0.6 \mathrm{fm}$ are plotted as a function of the temperature $T$.

Below $T_{c}, B_{>}(T)$ and $B_{<}(T)$ show a small $T$-dependence, implying that the thermal effect on the color screening factor is not high in the $T$ range investigated in the present analysis. $B_{>}(T)$ is approximately 2 times larger than $B_{<}(T)$, with this discrepancy indicating that the surrounding gluon configuration in the short $(R<0.6 \mathrm{fm})$ and long $(R \geq 0.6 \mathrm{fm})$ range regions, which are responsible for the screening mass, differ from each other in the confinement phase. While the gluon field stemming from a $q \bar{q}$ pair with a small separation $R$ yields a Coulomb type potential, it produces a linear confinement potential forming an in-between flux tube in the large $q \bar{q}$ separation region. A color leak to the flux tube leads to a mixture of random color components and yields a large screening mass. At $T=T_{c}$, both $B_{>}(T)$ and $B_{<}(T)$ show a sudden increase to much higher values, and this sudden change in the screening mass reflects the deconfinement phase transition, in which the color flux configuration around the $q \bar{q}$ pair exhibits a drastic change. Above $T_{c}$, $B_{>}(T)$ and $B_{<}(T)$ take more or less the same values, and the ratio of $B_{>}(T)$ to $B_{<}(T)$ is close to one. This is naturally expected, since the gluon field at $T>T_{c}$ produces a Yukawa-type potential for a $q \bar{q}$ pair over the whole $R$. $B_{<}(T)$ seems to gradually increase above $T_{c}$, which could be related to the increase of the screening mass in the Yukawatype potential in the deconfined phase.

The jump at $T=T_{c}$ can be found in both $B_{>}(T)$ and $B_{<}(T)$ also in the screening mass. $B_{<}(T)$ is much smaller than $B_{>}(T)$ below $T_{c}$, and an increase in $B_{<}(T)$ can be clearly observed. This order-parameter-like behavior of the color screening mass $B_{<}(T)$ in the short range region can again be understood as the sudden modification of the color flux configuration around the quarks. When the interquark distance $R$ is sufficiently small at a lower temperature, the color flux does not flow into a system around $q \bar{q}$ due to the color confinement, according to which only a color singlet $q \bar{q}$ state is physical. After the deconfinement transition occurs, a $q \bar{q}$ pair is able to even form a color-octet state, with the color largely leaking from the quarks throughout the system. This color leak leads to the sudden change of the color flux configuration, significantly increasing the color screening mass $B_{<}(T)$ across $T_{c}$.

Finally, here, we make a comparison between the screening masses obtained in our analysis and those estimated in previous works $[6,27,28]$. In Ref. [6], the Debye mass $m_{D}$ in an in-medium heavy quark potential was investigated, and researchers found that $m_{D} / T \sim 1.2$ at $T / T_{c}=1.5$. In Ref. [27], $m_{D} / T \sim 2.5$ was obtained from an analysis of the color singlet $q \bar{q}$ free energy $F_{1}$. The Debye mass $m_{E}$ estimated at 2-loop order shows that $m_{E} / T \sim 2.5$ in the temperature range we investigated here. The "color screening mass" $B$ obtained in the current analysis amounts to $B / T \sim$ 3.5 at $T / T_{c}=1.5$, which is significantly larger than the values found in previous studies. While previous estimates of the Debye mass $[6,27]$ were based on the heavy quark potential, our screening mass was extracted from the color correlation function $F(R)$. The relationship between the heavy quark potential and the color correlation function leaves room for discussion. Another possible origin of the deviation may lie in the channel investigated. While the target channel in Refs. [6,27] is a color singlet channel, our present calculation contains "contaminations" from the color octet states above $T_{c}$, which could have caused the difference.

\section{SUMMARY AND CONCLUDING REMARKS}

In this study, by means of an EE defined by reduced density matrices $\rho$ in a color space, we have investigated the color correlation of static $q \bar{q}$ systems at finite temperatures. We have adopted the standard Wilson gauge action on an anisotropic lattice and performed quenched calculations for density matrices. The gauge coupling is $\beta=5.75$ and the spatial volume is $L^{3}=24^{3}$. To directly evaluate the components of $\rho_{i j, k l}$, we have imposed the Coulomb-gauge on the gluon field. The temperatures we investigated range from 183 to $367 \mathrm{MeV}$.

Further, we have evaluated the $q \bar{q}$ correlation as a function of the $q \bar{q}$ separation $R$ based on the EE constructed from the reduced density matrix $\rho$ at finite temperatures. The Renyi entropy $S^{\text {Renyi- } \alpha}$ of the order $\alpha=2$ is adopted for the evaluation of EE. Below $T_{c}=285 \mathrm{MeV}$, we have found that the EE at each $q \bar{q}$ separation $R$ is barely affected by temperature, showing similar behavior to that at $T=0$. When a $q \bar{q}$ pair is located nearby, it forms a strongly correlated (color singlet) state $|\mathbf{1}\rangle$, and random color components (a random mixture of $|\mathbf{1}\rangle$ and $|\mathbf{8}\rangle$ ) appear, depending on $R$ in the large $R$ region, leading to color screening between 
quarks. As the temperature increases, such $q \bar{q}$ correlation is quickly lost during the phase transition at $T=T_{c}$ leading to an acute increase of the EE, which reflects the drastic modification of the gluon configuration at the phase transition. While the EEs for $R \geq 0.6 \mathrm{fm}$ reach almost the maximum value above $T_{c}$, the EEs for $R<0.6 \mathrm{fm}$ continue growing as $T$ increases, indicating that the $q \bar{q}$ correlation has a temperature dependence at $T>T_{c}$.

To understand the $R$ dependence of the $q \bar{q}$ color correlation in detail, we have extracted the color correlation function $F(R)$ based on the ansatz for the reduced density matrix $\rho$, in which $\rho$ is written by a sum of the color-singlet (correlated) state $|\mathbf{1}\rangle\langle\mathbf{1}|$ and random uncorrelated elements $|\mathbf{1}\rangle\left\langle\mathbf{1}|,| \mathbf{8}_{i}\right\rangle\left\langle\mathbf{8}_{i}\right|$ $\left(i=1, \ldots, N_{c}^{2}-1\right)$. In the analysis, we have extracted the "color screening mass" $B$ from the color correlation function $F(R)$, which represents the strength of the color screening. In particular, we have evaluated the short-range screening mass $B_{<}$and the long-range mass $B_{>}$defined in the ranges $R<0.6$ and $R \geq 0.6 \mathrm{fm}$, respectively. Below the phase transition temperature $T_{c}, B_{<}$and $B_{>}$differ in value, indicating the qualitative difference in gluon field profile between these two distinct ranges: one demonstrates a Coulomb-type gluon configuration and the other demonstrates a one-dimensional flux-tube profile. At the phase transition point $T=T_{c}$, the screening mass significantly and suddenly increases. Taking into account that the color screening mass encodes the surrounding gluon configuration around the $q \bar{q}$ pair, the enhancement of $B$ at $T_{c}$ should be related to the color flux delocalization caused by the deconfinement transition. Above $T_{c}, B_{<}$and $B_{>}$take similar values, consistent with the gluon profile, which gives a Yukawa-type screened potential over the entire $R$ range.

The EE and the screening mass values have been found to significantly change at the phase transition point, indicating that the EE properly detects the significant modification of the gluon configuration around the $q \bar{q}$ pair across the color deconfinement transition. In particular, the $\mathrm{EE}$ and the screening mass for small $R$ values behave like order parameters of the color confinement. Though these are not order parameters related to QCD symmetry, they demonstrate a clear physical meaning and can be good probes for the phase transition in finite $T / \mu$ systems.

In conclusion, the $q \bar{q}$ color correlations have been found to be well quantified by EEs at finite temperatures below and above $T_{c}$. These findings encourage us to adopt EE for the future study of the internal color structures of hadrons including multiquark systems [4] or the color structures of systems at finite $T / \mu$.

\section{ACKNOWLEDGMENTS}

This work was partly supported by Grants-in-Aid of the Japan Society for the Promotion of Science (Grants No. JP18K03617 and No. JP18H05407).
[1] J. Greensite, An Introduction to the Confinement Problem, Lecture Notes in Physics (Springer, New York, 2011), and references therein.

[2] G. S. Bali, K. Schilling, and C. Schlichter, Phys. Rev. D 51, 5165 (1995).

[3] T. T. Takahashi, H. Suganuma, Y. Nemoto, and H. Matsufuru, Phys. Rev. D 65, 114509 (2002).

[4] F. Okiharu, H. Suganuma, and T. T. Takahashi, Phys. Rev. D 72, 014505 (2005).

[5] O. Kaczmarek, F. Karsch, F. Zantow, and P. Petreczky, Phys. Rev. D 70, 074505 (2004); 72, 059903(E) (2005).

[6] Y. Burnier and A. Rothkopf, Phys. Rev. D 95, 054511 (2017).

[7] V. G. Bornyakov et al. (DIK Collaboration), Phys. Rev. D 70, 054506 (2004).

[8] T. T. Takahashi and Y. Kanada-En'yo, Phys. Rev. D 100, 114502 (2019).

[9] E. Itou, K. Nagata, Y. Nakagawa, A. Nakamura, and V. I. Zakharov, Prog. Theor. Exp. Phys. 2016, 061 B01 (2016).

[10] S. Aoki, T. Iritani, M. Nozaki, T. Numasawa, N. Shiba, and H. Tasaki, J. High Energy Phys. 06 (2015) 187.

[11] Y. Kanada-En'yo, Phys. Rev. C 91, 034303 (2015).

[12] T. Takayanagi, Classical Quantum Gravity 29, 153001 (2012).

[13] C. H. Bennett, H. J. Bernstein, S. Popescu, and B. Schumacher, Phys. Rev. A 53, 2046 (1996).
[14] P. Calabrese and J. L. Cardy, J. Stat. Mech. (2004) P06002.

[15] G. Vidal, J. I. Latorre, E. Rico, and A. Kitaev, Phys. Rev. Lett. 90, 227902 (2003).

[16] L. Amico, R. Fazio, A. Osterloh, and V. Vedral, Rev. Mod. Phys. 80, 517 (2008).

[17] R. Horodecki, P. Horodecki, M. Horodecki, and K. Horodecki, Rev. Mod. Phys. 81, 865 (2009).

[18] C. H. Bennett, D. P. DiVincenzo, J. A. Smolin, and W. K. Wootters, Phys. Rev. A 54, 3824 (1996).

[19] W. K. Wootters, Phys. Rev. Lett. 80, 2245 (1998).

[20] G. Vidal and R. F. Werner, Phys. Rev. A 65, 032314 (2002).

[21] G. Tiktopoulos, Phys. Lett. 66B, 271 (1977).

[22] J. Greensite and C. B. Thorn, J. High Energy Phys. 02 (2002) 014.

[23] A. Renyi, Probability Theory (Elsevier, New York, 1970).

[24] T. R. Klassen, Nucl. Phys. B533, 557 (1998).

[25] H. Matsufuru, T. Onogi, and T. Umeda, Phys. Rev. D 64, 114503 (2001).

[26] X. F. Meng, Y. Chen, and Y. B. Liu, Commun. Theor. Phys. 52, 665 (2009).

[27] O. Kaczmarek and F. Zantow, Phys. Rev. D 71, 114510 (2005).

[28] M. Laine, P. Schicho, and Y. Schröder, Phys. Rev. D 101, 023532 (2020). 